\title{
Adapting to remapped auditory localization cues: A decision-theory model
}

\author{
BARBARA SHINN-CUNNINGHAM \\ Boston University, Boston, Massachusetts
}

\begin{abstract}
This paper describes a model of adaptation to remapped auditory localization cues that is based on previous decision-theory models of psychophysical performance. The present model extends earlier work by explicitly assuming that past experience affects subject perception and by quantifying how training causes subjects' responses to evolve over time. The model makes quantitative predictions of total sensitivity, bias, and resolution for subjects involved in experiments investigating spatial auditory adaptation. One assumption of the model is that subjects cannot adapt to nonlinear rearrangements of localization cues, which is consistent with previous experimental reports in both audition (ShinnCunningham, Durlach, \& Held, 1998b) and vision (Bedford, 1993). The model assumes that, in spatial adaptation experiments, subjects learn to interpret a continuous internal decision variable differently than normal; they do not learn to associate discrete stimulus-response pairs. This view is consistent with previous analyses of results from experiments investigating adaptation to visual rearrangement, as well as with the McCullough effect in vision (Bedford, 1993, 1995).
\end{abstract}

There are many studies in which the effect of altering, or rearranging, sensory localization cues is investigated. In most such studies with vision, the perceptual effects of viewing the world through prisms that rotate the visual field are investigated. In the majority of such studies with audition, the question of how mean localization judgments change when the interaural axis is effectively rotated is investigated (see, e.g., Day \& Singer, 1967; Kalil \& Freedman, 1967; Mikaelian, 1969, 1972, 1974; Recanzone, 1998; Willey, Inglis, \& Pearce, 1937; Young, 1928). (For a review of auditory adaptation, see ShinnCunningham, Lehnert, Kramer, Wenzel, \& Durlach, 1997; for a review of adaptation studies in general, see Welch, 1978.)

In all such studies, naive subjects mislocalize sources in a direction predicted by the transformation imposed on the sensory stimuli reaching the eyes or the ears. If the subject is given appropriate training, however, these localization errors decrease with time. In most studies of sensory rearrangement, this training is achieved by allowing the subject to interact freely with the environment while observing the transformed sensory stimuli (e.g., by viewing their hand as they reach for a stimulus or by lis-

Portions of this work were presented at the 129th (May 1995) meeting of the Acoustical Society of America. This work was supported by AFOSR Grants 90-200, 93NL387, and 96NL145. Nat Durlach, Neil Macmillan, Bill Rabinowitz, Bruce Schneider, and an anonymous reviewer provided very thoughtful and insightful criticisms of earlier versions of this paper. Correspondence concerning this article should be addressed to B. Shinn-Cunningham, Department of Cognitive and Neural Systems, Boston University, 677 Beacon St., Room 311, Boston, MA 02215 (e-mail: shinn@cns.bu.edu).

- Accepted by previous editor, Myron L. Braunstein tening to sounds as they walk toward or away from a sound source). To date, models of adaptation to both auditory rearrangements and rearrangements in other modalities have set out to describe qualitatively the changes in response error that occur with training. In general, these models (see, e.g., Ebenholtz, 1970, 1973; Welch, 1978; Welch \& Warren, 1980) do not allow quantitative predictions of changes in response error, nor do they address how resolution might be affected by changes in the adaptive state of the subjects. Rather, these models enumerate the factors that are thought to influence localization errors.

Conversely, models of signal detection theory for onedimensional stimulus sets have been used to make quantitative predictions of both resolution and bias for a variety of stimulus types - for example, intensity perception (Braida \& Durlach, 1988; Braida et al., 1984; Durlach \& Braida, 1969; Luce, Green, \& Weber, 1976; Luce, Nosofsky, Green, \& Smith, 1982), auditory localization (Searle, Braida, Davis, \& Colburn, 1976), auditory lateralization (Koehnke \& Durlach, 1989), and speech perception (Macmillan, 1987; Macmillan, Braida, \& Goldberg, 1987; Macmillan, Goldberg, \& Braida, 1988). Such models allow quantitative predictions of resolution and/or bias for given experiments. However, these models generally assume that performance is stable and, thus, that resolution and bias have reached asymptotic values. These models do not describe the changes in resolution and bias over time that are seen in typical spatial rearrangement experiments.

This paper briefly reviews a set of experiments in which the effect of auditory rearrangement on mean localization judgments is investigated, as well as the effect on response bias and resolution of source locations. A previous psychophysical model (Durlach \& Braida, 1969) is extended in order to describe the empirical results of these 
"supernormal" localization studies. The model is then evaluated by comparing predicted and empirical results.

\section{SUMMARY OF EXPERIMENTS}

A number of experiments in which adaptation to remapped auditory localization cues was investigated have been reported previously (Durlach, Shinn-Cunningham, \& Held, 1993; Shinn-Cunningham, Durlach, \& Held, $1998 \mathrm{a}, 1998 \mathrm{~b})$. The goal of this work was to determine whether subjects could learn a new correspondence between spatial acoustic cues and reported azimuthal position that might enhance auditory spatial resolution (see Durlach et al., 1993). To this end, subjects were trained to identify the azimuthal location of an auditory source whose physical cues normally correspond to a different source position.

\section{Method}

Subjects were presented with auditory localization cues simulated over headphones, using head-related transfer functions (HRTFs). HRTFs are empirically determined filters that describe how to simulate (over headphones) the acoustic cues that would arise from a free-field sound source located at a specific angular location relative to the listener. In particular, the original source signal is convolved with a pair of filters (an HRTF filter pair) to generate a binaural signal whose interaural differences and spectral content are appropriate for the desired source at the simulated location. HRTF-based simulations are an increasingly common method for controlling spatial auditory cues in order to investigate the physiological and perceptual bases of spatial auditory perception (see, e.g., Brugge et al., 1994; Wightman \& Kistler, 1997). For a more complete discussion and review of these techniques, see Wenzel (1992) or Carlile (1996).

Normally, to simulate a source at azimuth $\theta$ and elevation $\phi$, one simply uses the empirically measured HRTF pair for that position, denoted in the frequency domain by $H(\omega, \theta, \phi)$, where $\omega$ corresponds to frequency. In our experiments, the correspondence between HRTFs and azimuth position was remapped so that $H^{\prime}(\omega, \theta, \phi)$, the HRTF pair used to simulate a source at position $[\theta, \phi]$, is given by

$$
H^{\prime}(\omega, \theta, \phi)=H\left(\omega, f_{n}(\theta), \phi\right),
$$

where $f_{n}(\theta)$, the family of mapping functions used to transform azimuth cues, is given by

$$
f_{n}(\theta)=\frac{1}{2} \tan ^{-1}\left[\frac{2 n \sin (2 \theta)}{1-n^{2}+\left(1+n^{2}\right) \cos (2 \theta)}\right] .
$$

With this transformation, a source whose position is "supposed" to be at $[\theta, \omega]$ is simulated by presenting the cues normally associated with a position of $\left[f_{n}(\theta), \phi\right]$. The parameter $n$ corresponds to the slope of the transformation at $\theta=0$. Normal localization cues are presented when $n=1$ [i.e., the function $f_{1}(\theta)$ is a straight line of slope one through the origin]. This mapping is shown in Figure 1 for the values of $n$ used in the experiments.

In order to determine whether the subjects could adapt to the remapping of HRTF cues, they were repeatedly tested over the course of experimental sessions lasting roughly $2 \mathrm{~h}$, first using the normal mapping ( $n=1)$ and then using an altered mapping $(n>1)$. At the end of the experimental session, testing with the normal mapping was repeated in order to look for aftereffects of the learned remapping. Every subject performed eight identical 2-h-long sessions over the course of 2-3 weeks. Data from each run within a session were combined with the data from the same run in the other sessions

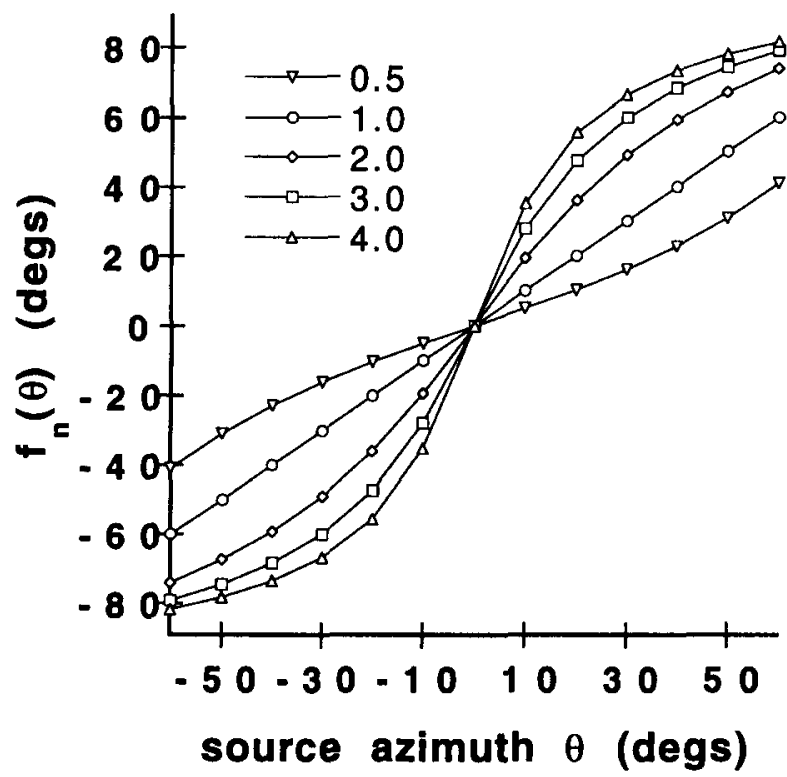

Figure 1. The family of functions $f_{n}(\theta)$ used to transform auditory localization cues. Using these transformations, a source from azimuth $\theta$ was synthesized, using the head-related transfer function that normally corresponded to the position $f_{n}(\theta)$.

to yield a total of 16 judgments of each source location from each subject in each run.

The subjects were seated inside a 5 -ft radius arc of 13 light bulbs, spaced every $10^{\circ}$ in azimuth from $-60^{\circ}$ to $+60^{\circ}$, which were labeled (left to right) with the numbers $1-13$. In each run, a 500 msec-long wideband click train was simulated from each of the possible locations exactly twice, in random order. The subjects were asked to identify the source azimuth corresponding to the simulated source position while facing straight ahead. For the experiments reported here (Experiments $F_{3}, F_{3 \text { mid }}, F_{2}, F_{4 a}$, and $F_{4 b}$ ), training was achieved by turning on the light at the correct, or nominal, location for $500 \mathrm{msec}$ after each response. Each subject performed 2 normal cue runs (using $n=1$ ), 30 altered cue runs (using $n>1$ ), followed by 8 posttraining runs. In all but one experiment, the posttraining runs all used normal cues (i.e., using $n=1$ ). In Experiment $F_{4 b}$, the 8 posttraining runs consisted of 4 runs in which $n=0.5$, followed by 4 normal cue runs.

\section{Results}

Previous reports (Shinn-Cunningham et al., 1998a) showed that the amount by which subject responses change during an experimental session depends primarily on the strength of the transformation (i.e., the value of $n$ ) and the range/number of source positions presented (ShinnCunningham et al., 1998a). The present analysis will focus on the results from experiments that differed only in these parameters. The transformation strengths and the number of source positions used in each experiment are summarized in Table 1 . In Experiments $\mathrm{F}_{3}, \mathrm{~F}_{2}$, and $\mathrm{F}_{4 \mathrm{a}}$, all 13 source positions were presented, and the transformation strength was varied (equal to 3,2 , and 4 , respectively, as denoted by the subscript). In Experiment $\mathrm{F}_{3 \text { mid }}$, the transformation strength equaled 3 , but only the middle 7 positions were presented (from -30 to $+30^{\circ}$ ). Fi- 
Table 1 Summary of the Experiments

\begin{tabular}{cccc}
\hline Experiment & $n$ & Subjects & Positions \\
\hline $\mathrm{F}_{3}$ & 3 & 5 & 13 \\
$\mathrm{~F}_{3 \text { mid }}$ & 3 & 4 & 7 \\
$\mathrm{~F}_{2}$ & 2 & 4 & 13 \\
$\mathrm{~F}_{4 \mathrm{a}}$ & 4 & 3 & 13 \\
$\mathrm{~F}_{4 \mathrm{~b}}$ & $4,0.5$ & 3 & 13 \\
\hline
\end{tabular}

Note- $n$ gives transformation strengths used, Subjects gives the number of subjects, and Positions shows the number of positions used in each experiment.

nally, Experiment $F_{4 b}$ was identical to Experiment $F_{4 a}$, except that, in the posttraining runs, the subjects were given both an inverse transformation $(n=0.5)$ and normal cues $(n=1.0)$, as was mentioned above. A more detailed explanation of the experimental methods and the rationale behind all of the chosen experimental conditions is given in Shinn-Cunningham et al. (1998a).

Various aspects of performance, including bias, resolution, and mean response, were estimated separately for each run of the experimental session as the subjects learned the remapping. In general, bias (a measure of mean response error in units of standard deviation) decreased as the subjects were exposed to the remapped cues, which is consistent with the subjects' learning the new mapping of physical cue to source location (ShinnCunningham et al., 1998a). However, some bias remained, even after performance had stabilized. In addition, the size of the localization bias was not uniform but varied with stimulus azimuth. The ability to resolve adjacent response locations changed abruptly when the remapping was introduced, as was expected. Resolution improved for stimuli that were physically more distinct with the remapped cues than with the normal mapping (sources in the front region, as seen in Figure 1) and decreased for stimuli that were more similar than normal (for $n>1$, this occurs for sources at the edges of the range). As the subjects adapted to the remapped cues, however, their ability to resolve the same physical stimuli showed an overall decrease, indicating that subjects confused adjacent stimuli more often after training than prior to training. Response bias and resolution are discussed in greater detail when compared with predictions from the adaptation model (see Figures 5 and 6, respectively).

\section{Analysis and Discussion}

Previous analysis of changes in mean response (ShinnCunningham et al., 1998b) showed that subjects did not adapt exactly to the transformations shown in Figure 1. Over time, mean responses did change, but these changes were consistent with subjects' learning a linear remapping of azimuthal cues instead of the nonlinear remapping that was actually imposed. The observed behavior implies that, during run $r$, the mean perceived position of a source whose nominal location equals $\theta$ is given by

$$
p(\theta, r)=k(r) f_{n}(\theta)
$$

where $k(r)$ is a dimensionless slope that varies with run $r$ and $f_{n}(\theta)$ is the location normally associated with the localization cues presented (see Equation 2). For normal cue presentations, $n=1$ and $f_{n}(\theta)=\theta$. Thus, for normalcue runs, the mean perceived azimuthal position of a stimulus equals the nominal location $(\theta)$ scaled by $k(r)$. For naive subjects, prior to any training with the rearranged acoustic cues, $k(r)$ was roughly equal to one, as was expected. That is, for the initial normal-cue runs at the beginning of each session, $p(\theta, r)=\theta$. Subjects heard sources at the position normally associated with the HRTFs employed, and this location was the nominal location. For the altered-cue conditions, the mean perceived azimuth equals $f_{n}(\theta)$ (the azimuth to which the physical stimulus normally corresponds) scaled by the slope $k(r)$. According to Equation 3, complete adaptation to the transformed cues does not occur, because there is no value of $k(r)$ for which $p(\theta, r)$ equals $\theta$ for all possible locations, unless $n$ equals one.

Equation 3 implies that changes in perceived location depend only on changes in $k(r)$, the slope relating mean perceived location to the normal azimuthal locations of the stimuli. The effect of the exposure to the altered cues is to change the slope $k(r)$. When a transformation was first introduced (i.e., $n$ was changed from 1 to a value $n$ $>1$ ), the subjects interpreted the new set of physical cues as they normally would (i.e., slope, $k$, near unity), resulting in mean errors in their location judgments. As the subjects were trained, $k(r)$ decreased gradually, causing the average size of the judgment errors to decrease. However, since the nominal responses are not linearly related to the normal locations corresponding to the physical cues presented, systematic response errors persisted.

This is shown schematically in Figure 2, which plots the perceived position as a function of $f_{n}(\theta)$, the location

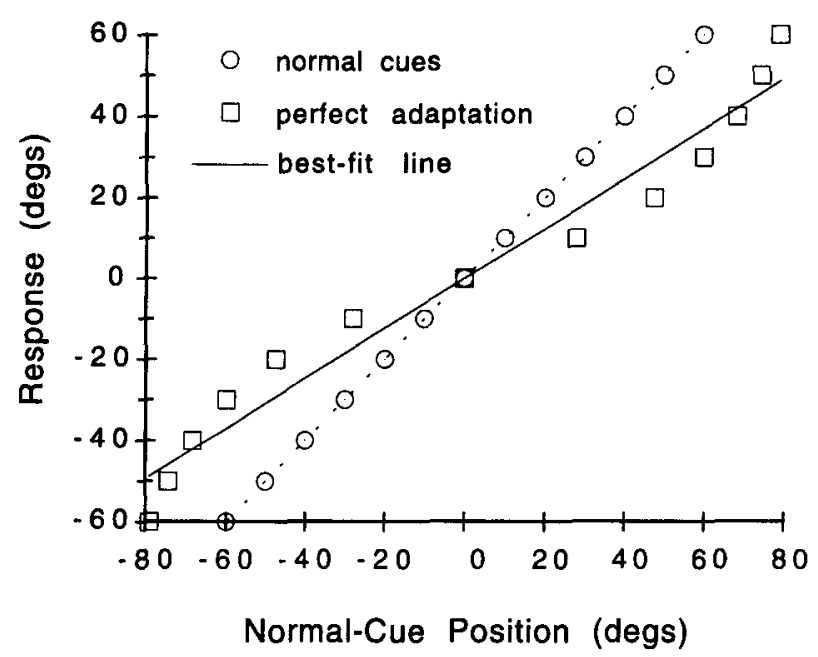

Figure 2. Schematic representation of the observed constraint on adaptation. The abscissa represents $f_{n}(\theta)$, the location normally associated with the stimulus whose nominal location is $\theta$. The ordinate represents the mean response given by the subject. In normal-cue runs, the correct responses for the cues presented (open circles) fall on a line of slope one. In altered-cue runs, the correct responses (open squares) are a nonlinear function of $f_{n}(\theta)$. The solid line represents how subjects actually adapt to this nonlinearity. 
normally associated with the physical stimulus presented. The open circles in the figure represent the ideal mapping of physical cue to position during normal-cue runs. The open squares show the ideal mapping for runs when $n=3$. Note that in both cases, there are 13 points plotted, corresponding to the 13 available responses $(-60$, $-50, \ldots$ to 60 ) along the $y$ dimension. However, the normal-cue locations at which these values are plotted along the $x$ dimension are different for normal and altered cues, since the physical stimuli presented depend on the imposed transformation. Prior to adaptation, subjects interpret normal cues correctly with a slope $k=1$ (the dashed line connecting the open circles). When the physical stimuli are transformed, the physical stimuli span a range normally associated with locations from roughly $-80^{\circ}$ to $+80^{\circ}$. In addition, the nominal responses are no longer linearly related to the locations normally associated with the physical stimuli presented. When the transformation is first imposed, subjects interpret the stimuli in the normal way (i.e., $k$ is approximately equal to 1 ) and, therefore, make large localization errors. As subjects adapt, the slope of the mapping between stimulus and perceived position decreases so as to approximate the nonlinear transformation. Averaged across all source positions, the mean response error is reduced when the slope decreases, although systematic errors remain as a function of position. When the normal cues are reinstated at the end of the experiment, $k(r)$ increases back toward $k=1$.

The observed changes in slope $k(r)$ were roughly exponential, from an initial value toward an asymptotic value denoted by $T$. Mathematically, if the transformation changed in run $r_{c}, k(r)$ is given by

$$
k(r)=T+\left[k\left(r_{c}-1\right)-T\right] e^{-b\left(r-r_{c}\right)}
$$

for $r \geq r_{c}$ (and up until the next change in cue transformations), where $b$ is a parameter (in units of run ${ }^{-1}$ ) governing the rate of change in $k(r)$, and $k\left(r_{c}-1\right)$ is the slope value prior to the change in the auditory cues. The rate of change in $k(r)$ was roughly independent of how the cue transformation changed; all the data were fit well using $b=0.84$ run $^{-1}$ (Shinn-Cunningham et al., 1998b).

The adaptation rate, $b$, was independent of the change in transformation; however, $T$, the asymptotic value of $k(r)$, varied with $n$ (the strength of the transformation), as well as with the range of positions presented in each experiment. In particular, it was shown that, for all experiments, $T$ was roughly equal to $k_{A}$, the value of $k(r)$ that minimizes the mean-squared difference between $k(r) f_{n}(\theta)$ and $\theta$, the nominal position of the source. (The solid line in Figure 2 shows the best-fit line for the example with $k_{A}=0.61$.) For runs in which the cues are nonlinearly transformed, the mean response errors (across the stimuli presented) decrease to the minimum achievable, given the linear constraint of Equation 3 .

Adaptation to the nonlinear transformation is summarized by an exponential change in the slope $k(r)$. The slope exponentially approaches the best linear approximation of the transformation presented to the subjects. The value of the slope $k(r)$ summarizes the adaptive state of the subject during the course of the experiment. This finding implies that there are limits on the types of auditory spatial cue transformations that subjects can accommodate and that subjects may not be able to adapt to nonlinear transformations of localization cues. This type of constraint is similar to constraints that have been observed in visual spatial rearrangement experiments (Bedford, 1993, 1995).

\section{THE PRELIMINARY MODEL OF AUDITORY ADAPTATION}

The preliminary model of adaptation to remapped auditory localization cues is based on the preliminary model of intensity perception by Durlach, Braida, and associates (Braida \& Durlach, 1972, 1988; Durlach \& Braida, 1969). This model focused primarily on predicting resolution results for various experimental paradigms measuring intensity perception. However, the underlying assumptions of the model are easily applied to experiments investigating resolution in other stimulus dimensions, including stimulus location.

\section{The Preliminary Model of Intensity Perception}

In the preliminary model of intensity perception (described fully in Durlach \& Braida, 1969), every stimulus $I$ gives rise to an internal sensation $Y$, which is a Gaussian random variable with mean $\alpha(I)$ and variance $\beta^{2}$. This sensation is then further transformed into a decision variable along an internal axis to produce a Gaussian random variable $Q$, which has mean $\alpha(I)$ and variance $\beta^{2}+\gamma^{2}$. In this model, internal noise arises from two independent sources. Sensation noise (with variance $\beta^{2}$ ) depends only on the stimulus presented and, therefore, causes a fixed limit on the best performance that can be achieved in any experiment. Memory noise (with variance $\gamma^{2}$ ) affects the transformation from the sensation $Y$ to the internal representation of the decision variable $Q$ and depends on the type of experiment. For singleinterval experiments such as those in the present study, memory noise is assumed to be proportional to the total range of stimuli presented in the experiment and is termed context-coding noise. Thus, $\gamma^{2}=G^{2} R^{2}$, where $G$ is a constant, $R=\alpha\left(I_{\max }\right)-\alpha\left(I_{\min }\right)$, and $I_{\max }$ and $I_{\min }$ are equal to the extreme values of the stimuli used in the experiment. The addition of context-coding noise allows the model to account for the fact that subjects may confuse two stimuli in large-range tasks (such as identification tasks), even when the same stimuli are perfectly resolvable in tasks in which the range is small (such as in JND-type tasks).

The preliminary intensity perception model further assumes that subject responses are based on the value of the decision variable $Q$ using a Thurstonian decision model. With these assumptions, the decision axis along 
which $Q$ falls is assumed to be divided into $n$ contiguous regions by $n+1$ criteria $\left\{C_{j}\right\}$ (with $C_{0}=-\infty$ and $C_{n+1}=$ $\infty)$. Each region corresponds to one of the $n$ possible responses for the experiment being performed. In a single interval task, the means of the underlying probability distributions $P\left(Q \mid I_{i}\right)$ depend only on the stimulus presented $\left(I_{i}\right)$, and the variance (which is constant, independent of the stimulus value) depends only on the range of stimuli used in the experiment. The underlying discriminability between two stimuli $I_{i}$ and $I_{j}$ in a one-interval experiment can then be written as

$$
d_{R}^{\prime}\left(I_{i}, I_{j}\right)=\frac{\alpha\left(I_{i}\right)-\alpha\left(I_{j}\right)}{\sqrt{\beta^{2}+G^{2}\left[\alpha\left(I_{\max (R)}\right)-\alpha\left(I_{\min (R)}\right)\right]^{2}}},
$$

where $R$ is the range of stimuli used in the experiment, $\beta$ and $G$ are constants, $I_{\max (R)}$ and $I_{\min (R)}$ are the maximum and minimum stimulus intensities in the range $R$, and $\alpha(\bullet)$ is a monotonic transformation that relates the physical stimulus values to values along an internal decision axis. It can be seen from Equation 5 that the ability to resolve two stimuli depends only on the range of stimuli used in the experiment and the stimulus values.

\section{An Internal Representation of Azimuth}

Many of the ideas used in developing the preliminary model of intensity perception have been used to model perception of other stimulus dimensions, including speech (Macmillan, 1987; Macmillan et al., 1987; Macmillan et al., 1988), auditory localization (Searle et al., 1976), and auditory lateralization (Koehnke \& Durlach, 1989). However, no previous work quantified how auditory localization resolution depends on absolute position. In the preliminary model of intensity perception, relative sensitivity is taken into account by the mapping function $\alpha(\bullet)$, which is responsible for converting values from the stimulus space to a dimension in which distance is directly proportional to discriminability between stimuli. For the present model, the function $\alpha(\theta)$ was roughly fit using data from an early free-field study (Mills, 1958) of the minimum audible angle (MAA). Details in the methodology for fitting $\alpha(\theta)$ are given in Shinn-Cunningham (1994). The resulting function is given by

$$
\alpha(\theta)=\operatorname{sign}(\theta)\left(1-e^{-\mathrm{a}|\theta|}\right),
$$

where $a=0.02 \mathrm{deg}^{-1}$. The form of this function reflects the fact that the MAA is small around a reference position of $0^{\circ}$ azimuth and grows rapidly for reference locations to either side of the listener. As a result of this mapping, when a pair of stimuli at $\theta^{\circ}$ and $\theta+\Delta$ deg are mapped to a pair of probabilistic distributions of the internal decision variable, the distance between the means of the distributions decreases as the magnitude of $\theta$ grows. Since the model assumes that the standard deviation of the noise in the internal decision variable is independent of the stimulus value, the model predicts that sources to the side are less easily resolved than are sources near zero azimuth.

\section{Extending the Model to Adaptation Paradigms}

In order to account for changes in performance that occur in adaptation paradigms, the preliminary intensity perception model of Durlach and Braida (1969) is extended in two important ways: (1) The effective range $R$ depends both on the stimuli being presented at a given point in time and on the past experience of the subject; $R$ is no longer determined exclusively by the stimuli used in an experiment; and (2) the criteria $\left\{C_{j}\right\}$ are not assumed to be optimally positioned for the given stimuli; their placement depends on the past experience of the subject.

Intuitively, it is unlikely that subjects immediately change their behavior when the physical stimuli change abruptly. Therefore, when the stimulus range changes in the adaptation experiment, it is unlikely that the memory noise in the underlying decision space will be determined by the range of the physical cues being presented immediately after the change. Rather, the model predicts that the range of cues that the subject expects to hear determines the internal decision noise. Just after a change in stimulus range, the expected or effective range should roughly equal the range of cues presented in the previous run (assuming that performance was stable prior to the change in cues). Over time, the effective range should approach the actual range of cues being presented. Similarly, the model assumes that, just after the remapping is introduced, subjects make their response decisions on the basis of the positions of the decision-axis criteria prior to the change. Over time, the criteria shift to new positions, more appropriate for the present stimulus-response mapping. The adaptation model assumes that both of these gradual changes (in the effective range and in the placement of the decision criteria) are governed by changes in an internal mapping maintained by the subject. This internal representation describes how physical stimuli are related to the available range of responses at a given point in time.

The model assumes that the slope $k(r)$, which relates the normal-cue position of a physical stimulus to the mean subject response, summarizes the state of the posited internal map throughout the experiment. During the adaptation experiments, a subject knows that the range of possible responses is restricted, usually from $-60^{\circ}$ to $+60^{\circ}$. During a given run $r$, however, the subject maps that same range of responses to a different range of physical cues, as described by Equation 3. Thus, in run $r$, the minimuin physical cue that the subject expects is equal to

$$
\frac{\theta_{\min }}{k(r)},
$$

and the maximum physical cue he expects equals

$$
\frac{\theta_{\max }}{k(r)}
$$

(where $\theta_{\min }$ and $\theta_{\max }$ are the azimuthal angles of the minimum and the maximum allowed responses, respectively). These physical cue values correspond to values along the internal decision axis of 


$$
\alpha\left[\frac{\theta_{\min }}{k(r)}\right]
$$

and

$$
\alpha\left[\frac{\theta_{\max }}{k(r)}\right],
$$

respectively. Thus, in run $r$, the model assumes that $R_{\text {eff }}$, the effective stimulus range in run $r$, is given by

$$
R_{e f f}=\alpha\left[\frac{\theta_{\max }}{k(r)}\right]-\alpha\left[\frac{\theta_{\min }}{k(r)}\right] .
$$

Similarly, the subject expects to hear a set of physical stimuli that are different from the actual stimulus values presented. In particular, from Equation 3, the subject expects the stimulus $\theta_{j}$ to map to physical stimulus

$$
\frac{\theta_{j}}{k(r)} \text {. }
$$

Thus, in the underlying decision space, the subject expects the mean value of the stimulus whose nominal response is $\theta_{j}$ to equal

$$
\alpha\left[\frac{\theta_{j}}{k(r)}\right] .
$$

However, the stimulus whose nominal location is $\theta_{j}$ is the same physical stimulus whose normal location is $f_{n}\left(\theta_{j}\right)$. Thus, in the underlying decision space, the actual stimulus has a mean value of $\alpha\left[f_{n}\left(\theta_{j}\right)\right]$. If the subject tries to place decision criteria ideally, he will place them midway between the expected mean locations, not midway between the mean locations of the values actually presented. The model predicts that, in run $r$, the decision-axis criterion $C_{j}$ will equal

$$
C_{j}(r)=\frac{1}{2}\left\{\alpha\left[\frac{\theta_{j}}{k(r)}\right]+\alpha\left[\frac{\theta_{j+1}}{k(r)}\right]\right\},
$$

whereas the optimal placement (independent of run) is given by

$$
C_{j}^{\mathrm{opt}}=\frac{1}{2}\left\{\alpha\left[f_{n}\left(\theta_{j}\right)\right]+\alpha\left[f_{n}\left(\theta_{j+1}\right)\right]\right\} .
$$

Figure 3 illustrates how the internal decision variable and the criteria change in the model for an experiment similar to Experiment $F_{3 m i d}$ (in which seven stimuli, ranging from $\theta=-30^{\circ}$ to $\theta=30^{\circ}$, were presented). In the figure, panel a shows the distributions of the internal decision variable that would arise for the seven normal-cue stimuli presented to a naive subject. In this condition, there is no discrepancy between the stimulus values expected by the subject and the values presented, so the subject places his criteria (the dashed vertical lines) at optimal locations along the decision axis. The variance in the distributions is determined by the internal decision noise that, in turn, depends on the effective range. For this condition, there is no discrepancy between the effective range and the actual range of the stimuli presented. The second panel shows what occurs when the transformed cues are first introduced: Criteria are located at their optimal locations for the normal cues, not for the transformed cues. In addition, the internal sensation noise is determined by the range spanned by the normal-cue stimuli, not by the actual range of the stimuli now being presented. Panel $\mathrm{c}$ shows the effects of adaptation. Criteria are shifted in the correct direction but are not positioned optimally, since the subjects adapt to a linear approximation of the transformation employed in the experiments. In addition, memory noise grows, reflecting the fact the effective range grows as subjects learn to expect a larger range of stimuli. Finally, panel d shows what occurs for normal-cue stimuli following training. The decision criteria are at their adapted locations, causing bias in the direction opposite that initially introduced with the cue transformation. Similarly, memory noise is determined by the range expected with transformed cues and is, thus, larger than in the pretraining, normal-cue condition.

\section{MODEL PREDICTIONS}

\section{Total Cumulative Sensitivity}

There are two free parameters in the model: $\beta$, the standard deviation of the sensation noise, and $G / \beta$, the relative importance of the context-coding noise as compared with the sensation noise. Model parameters were estimated by examining the total cumulative sensitivity, $\Delta^{\prime}$. Total cumulative sensitivity measures the total distance along the internal decision axis between the mean values of the minimum and the maximum stimuli used in an experiment, measured in units of the standard deviation in the decision variable. ${ }^{1}$ Thus, $\Delta^{\prime}$ is given by

$$
\begin{aligned}
\Delta^{\prime} & =\frac{\alpha\left[f_{n}\left(\theta_{\max }\right)\right]-\alpha\left[f_{n}\left(\theta_{\min }\right)\right]}{\sqrt{\beta^{2}+G^{2} R(r)^{2}}}= \\
& =\frac{\alpha\left[f_{n}\left(\theta_{\max }\right)\right]-\alpha\left[f_{n}\left(\theta_{\min }\right)\right]}{\sqrt{\beta^{2}+G^{2}\left\{\alpha\left[\frac{\theta_{\max }}{k(r)}\right]-\alpha\left[\frac{\theta_{\min }}{k(r)}\right]\right\}^{2}}} .
\end{aligned}
$$

For each subject, the values of $\beta$ and $G / \beta$ that minimized the mean-square difference between predicted values of $\Delta^{\prime}$ and the individual data were estimated. These values were averaged across subjects. The resulting values are $\beta=0.06$ and $G / \beta=1.43$ (nearly identical results were found using a minimum mean-square error criterion in $\log \Delta^{\prime}$ space). It should be noted that for the experiments considered here, the model is not extremely sensitive to 
a) Normal cues, pre-training

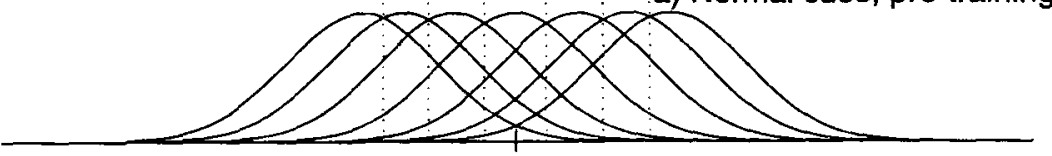

b) Altered cues, pre-training

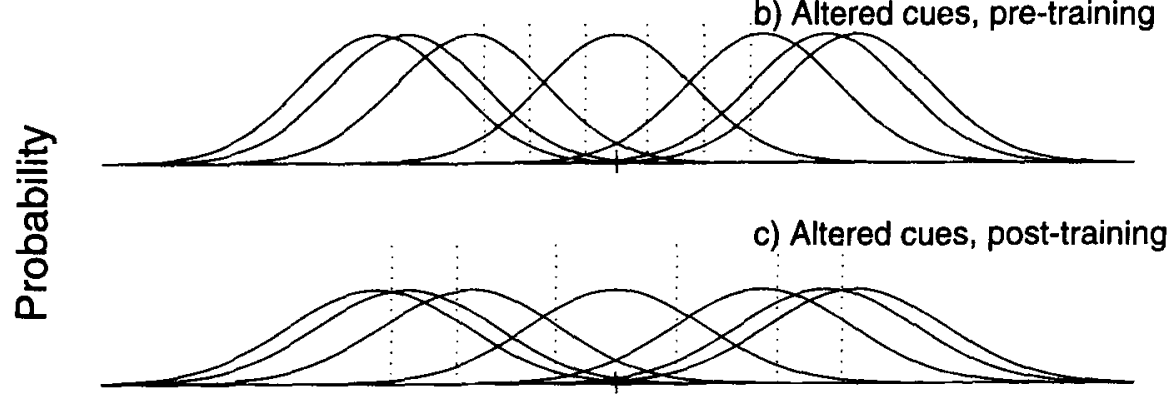

d) Normal cues, post-training

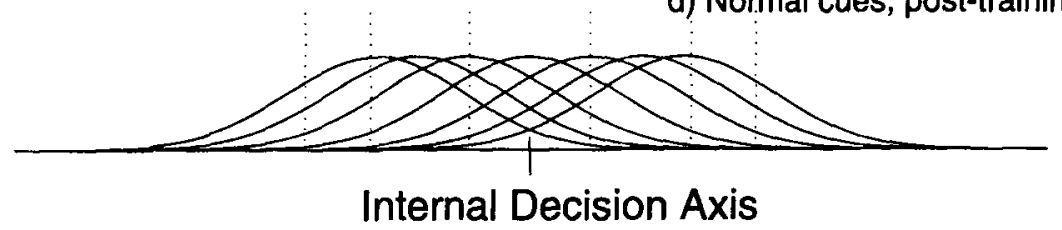

Figure 3. Representation of the internal decision axis for a seven-alternative, forced-choice task similar to that in Experiment $F_{3 \text { mid }}$ (in order to emphasize model behavior, transformation strength $n$ is equal to 6). (a) initial run with normal cues; (b) initial run with altered cues; (c) final run with altered cues; and (d) initial, posttraining run with normal cues. In each panel, the probability distributions of the internal decision variable corresponding to each of seven possible stimulus values are plotted. The criteria dividing the decision axis into seven distinct regions are shown by the dashed vertical lines.

the exact values of $\beta$ and $G / \beta$. In particular, for the experiments considered, in which

and

$$
\theta_{\max }=-\theta_{\min }
$$

$$
\beta^{2} \ll 4 G^{2} \alpha^{2}\left[\frac{\theta_{\max }}{k(r)}\right],
$$

Equation 10 can be approximated by

$$
\Delta^{\prime} \approx \frac{\alpha\left[f_{n}\left(\theta_{\max }\right)\right]}{\beta\left(\frac{G}{\beta}\right) \alpha\left[\frac{\theta_{\max }}{k(r)}\right]} .
$$

Thus, as long as the product of the estimated parameters $\beta$ and $G / \beta$ is constant, predictions are roughly comparable for the identification experiments considered. This product varied from subject to subject, consistent with the idea that different subjects have different overall levels of performance (i.e., different levels of internal noise). Despite the fact that there are differences across subjects (and that different subjects performed each experiment), only the average parameter values are used for all subsequent model predictions. This choice was made, in part, because all the data to which the predictions will be com- pared are across-subject averages and, in part, because it reduces the number of free parameters in the model. Thus, the model predictions presented in this paper represent the performance expected of a typical subject, rather than of the subjects who performed any specific experiment.

Figure 4 plots predictions of total cumulative sensitivity (using Equation 9 and the best-fit values of $\beta$ and $G / \beta$ ) and the empirically determined total cumulative sensitivity as a function of run. In each panel, the filled circles show the predicted values of $\Delta^{\prime}(r)$ for the corresponding experiment. Also plotted is the mean value of the empirical estimates of $\Delta^{\prime}$, averaged across subjects, plus or minus one standard deviation.

As was stated above, intersubject differences in the magnitude of $\Delta^{\prime}$ are large within each experiment (a difference for which the model could account by allowing the internal noise to vary from subject to subject). This intersubject variability accounts, at least in part, for discrepancies between the estimated and the actual magnitude of $\Delta^{\prime}$ in the different experiments (e.g., the results for Experiments $F_{4 a}$ and $F_{4 b}$, which present identical stimuli for Runs 1-32, show large differences in mean values of $\Delta^{\prime}$ for these same runs). Overall, the observed level of performance is better than predicted in Experi- 
ment $F_{3}$, worse in Experiment $F_{4 a}$, and in good agreement for the remaining three experiments.

Even more important than the overall magnitude of the predictions are the details of how $\Delta^{\prime}$ changes with run. In all cases, whenever the localization cues change, there are two effects: an immediate effect on the actual range of stimuli being discriminated (numerator in Equation 10) and a gradual growth of the effective stimulus range (and in the internal noise in the model; i.e., the denominator in Equation 10). In the present experiments, when the cue transformation is first introduced (e.g., in Run 3 for all experiments), the physical cue range increases slightly, causing an immediate increase in predictions of $\Delta^{\prime}$. These predicted changes are not obvious in the empirical data; however, the predicted changes are small, relative to the variability in the underlying data. As subjects adapt, the internal memory noise increases, and there is a concomitant decrease in predictions of $\Delta^{\prime}$ until performance stabilizes and reaches a steady-state value.

Steady-state predictions of $\Delta^{\prime}$ are approximately equal across all the experiments. This result is easily understood by examining the numerator and denominator in Equation 11. For the reported experiments (in which internal noise is dominated by context-coding noise), $\Delta^{\prime}$ is roughly proportional to the ratio of the actual stimulus range divided by the expected range. Although the expected range varies with $k(r)$, its steady-state value (when $k(r)$ has approached its asymptote) is roughly equal to the actual range. Thus, for all the experiments reported here, $\Delta^{\prime} \approx G^{-1}$ in the steady-state.

When the cue transformation changes at the end of the session, predicted values of $\Delta^{\prime}$ change again. In particular, in all the experiments, the actual range of stimuli (the numerator in Equation 10) decreases abruptly between Runs 32 and 33. At the same time, the internal noise (the denominator in Equation 10) begins to decrease toward its asymptotic value when the cue transformation changes. In most experiments, there is little discernable change in the empirical values of $\Delta^{\prime}$ between Runs 32 and 33 . However, in two experiments, empirical values of $\Delta^{\prime}$ showed a significant change between Runs 32 and 33: In Experiment $F_{3 m i d}, \Delta^{\prime}$ was significantly lower for normal-cue runs $(n=1)$ following adaptation to transformed cues $(n=3)$, and in Experiment $\mathrm{F}_{4 \mathrm{~b}}, \Delta^{\prime}$ was significantly smaller for the runs in which $n=0.5$ following training with $n=4$. The model predicts similar changes for both experiments. In the model, these decreases arise because the range of physical cues presented to the subject decreases drastically with the change in cue transformation. At the same time, the starting value of the effective range is relatively large immediately after the change in cues (i.e., the denominator in Equation 10 is initially large). The model predicts qualitatively similar effects for Experiments $F_{3}, F_{2}$, and $F_{4 a}$. However, for these experiments, the percentage change in range is much smaller, and the predicted effect is small in comparison with the intrasub-

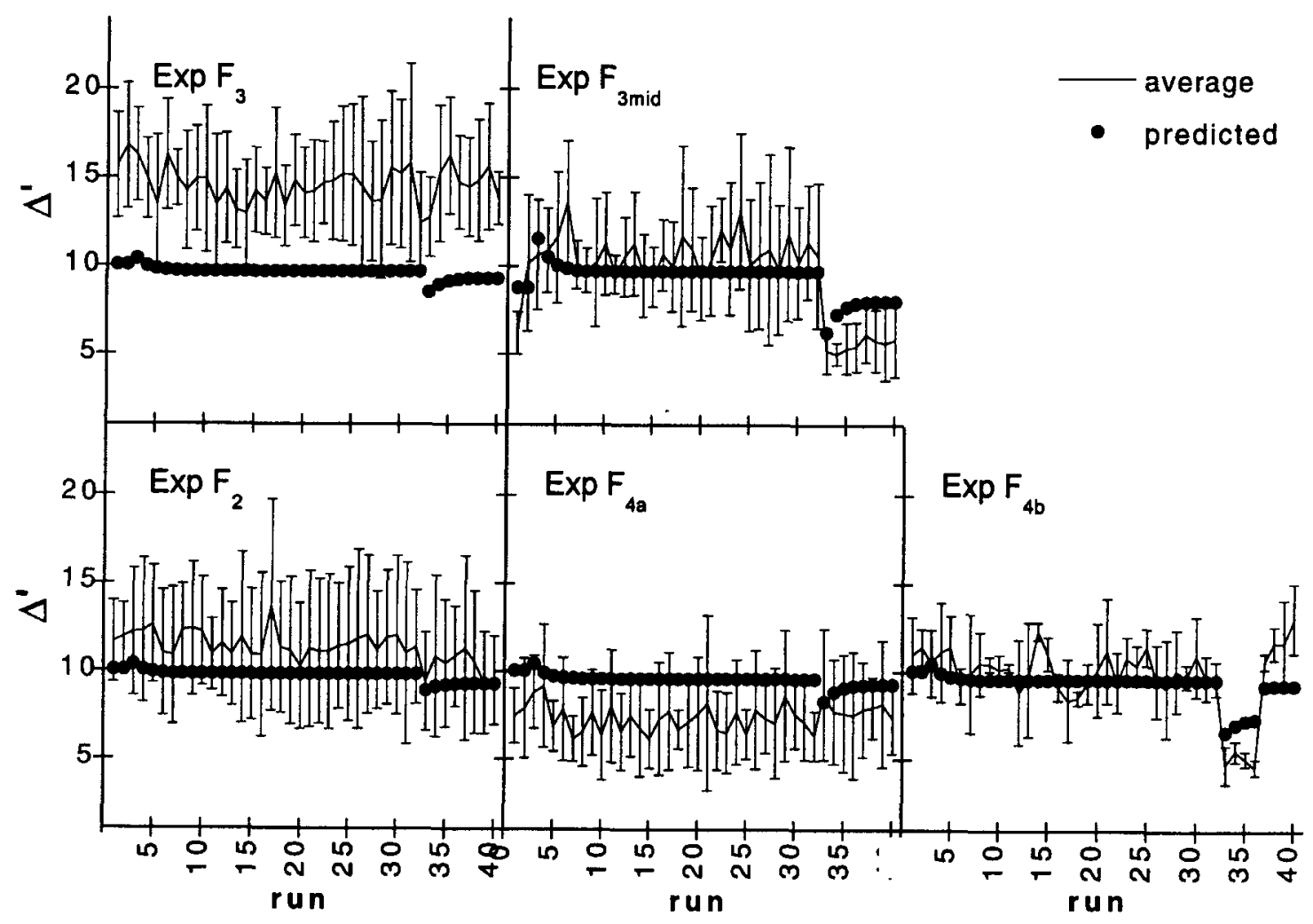

Figure 4. Estimated and predicted $\Delta^{\prime}$ as a function of run. 
ject variability (i.e., as compared with the random, nonsystematic variations in estimates of $\Delta^{\prime}$ for each subject as a function of run). In Experiments $F_{3 \text { mid }}$ and $F_{4 b}$, the magnitude of the predicted decrease in $\Delta^{\prime}$ at Run 33 was slightly smaller than that seen in the actual data; however, in both cases, the fit was relatively good.

Finally, in Experiment $F_{4 b}$, the model predicts an abrupt increase in $\Delta^{\prime}$ in Run 37, as compared with Run 36 . This prediction arises because the range of stimuli increases between these runs, causing an immediate increase in the numerator and a slow decrease in the denominator in Equation 10. The empirical results also show an immediate increase in $\Delta^{\prime}$ in Run 37 ; however, the increase is larger than that predicted by the model.

\section{Response Bias}

The bias, $B_{j}$, is one of the performance metrics investigated in a previous paper (Shinn-Cunningham et al., 1998a). ${ }^{2} B_{j}$ is equal to the difference between the optimal and the actual placement of the $j$ th decision criteria. Bias is related to absolute localization accuracy; when criteria are placed optimally, the probability of responding correctly on a given trial is maximized (if all the stimuli are equally likely), and any response error is due to in- ternal noise in the decision variable. Thus, from Equations 8 and 9 , bias in the placement of the $j$ th criterion is given by

$$
\begin{aligned}
& B_{j}(r)=\frac{C_{j}^{\mathrm{opt}}-C_{j}(r)}{\sqrt{\beta^{2}+G^{2} R(r)^{2}}} \\
& =\frac{\frac{1}{2}\left\{\alpha\left[f_{n}\left(\theta_{j}\right)\right]+\alpha\left[f_{n}\left(\theta_{j+1}\right)\right]\right\}-\frac{1}{2}\left\{\alpha\left[\frac{\theta_{j}}{k(r)}\right]+\alpha\left[\frac{\theta_{j+1}}{k(r)}\right]\right\}}{\sqrt{\beta^{2}+G^{2}\left\{\alpha\left[\frac{\theta_{\max }}{k(r)}\right]-\alpha\left[\frac{\theta_{\min }}{k(r)}\right]\right\}^{2}}}
\end{aligned}
$$

where $\theta_{j}$ is the nominal source position for the $j$ th stimulus.

Empirical results and model predictions of bias are shown in Figure 5: the left half of each panel shows empirical estimates of bias for sources to the left of center, and the right half of each panel shows the model predictions for sources to the right of center. The model parameters used in this figure (and in resolution predictions, shown in Figure 6) are identical to the values chosen to fit $\Delta^{\prime}$ in Figure 4. The experimental bias was first esti-

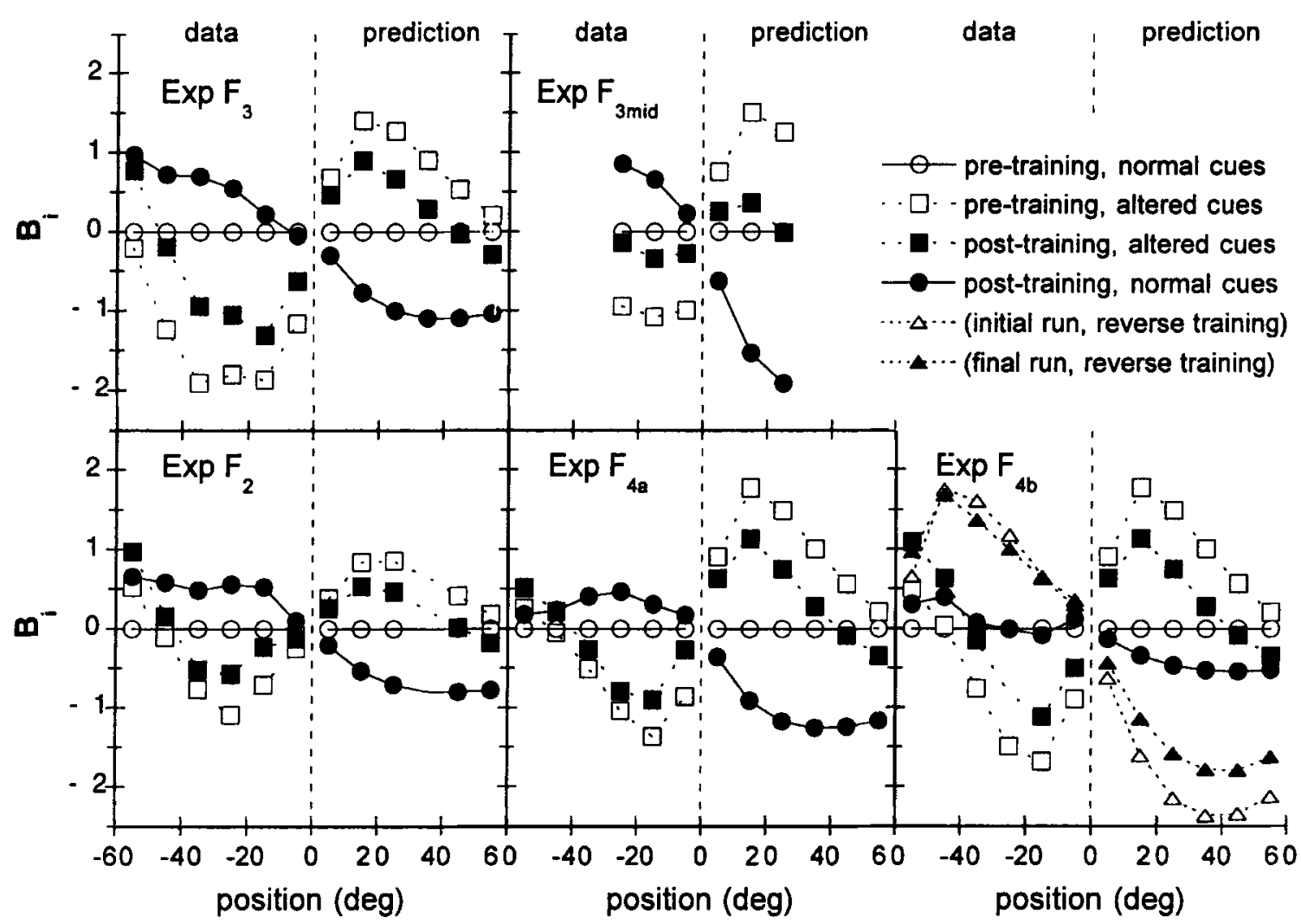

Figure 5. Estimated and predicted bias $B_{i}$ as a function of position. In each panel, the experimental results, plotted on the left side of each panel, were found by assuming that performance was symmetric about the midline. The predictions from the model are shown on the right side of each panel. To the extent that the left and right halves of each panel are symmetric about the line $y=-x$, the model fits the experimental results. 
mated individually for each subject. ${ }^{3}$ These values were then normalized by subtracting the average bias in the first normal-cue run to remove any bias effects not due to training. The normalized estimates of bias were then averaged across all subjects and then further collapsed by assuming left-right symmetry to yield the data plotted in Figure 5.

Focusing first on the experimental results, we see that, because of the normalization performed, initial bias with normal cues is identically equal to zero for all positions. Without this normalization, some initial bias was seen for many subjects; however, the magnitude of this initial bias was small relative to the bias seen in all of the other runs. When transformed cues are first introduced, a large bias results in the direction expected on the basis of the transformation (i.e., subjects tend to place the criteria too close to the zero-azimuth stimulus location) ${ }^{4}$ The bias is reduced with exposure to the altered cues to roughly $50 \%$ of the initial bias observed. When returning to normal cues, there is a negative aftereffect in the direction opposite the initial bias. The magnitude of the initial bias and negative aftereffect appear to be directly related to the strength of the transformation, with greater initial bias and aftereffect for more extreme transformations. In Experiment $F_{4 b}$, the bias for the initial run with the transformation $n=0.5$ resulted in a larger reverse bias than was seen using normal cues $(n=1)$, as was expected. By the final run with these cues, the effect diminished. The effect of this training on the bias in Run 37 (using normal cues) was to decrease the negative aftereffect seen when returning to normal cues following exposure to the "opposite" transformation, $n=0.5$.

The predictions for all experiments are in good agreement with the data (to the extent that the panels are symmetric about the line $y=-x$, the data and the model predictions are equal). A large bias is evident with the first altered-cue run, which decreases by the end of the training. A negative aftereffect occurs when first tested with normal cues after the altered-cue exposure. In the experimental data, the size of the bias and negative aftereffect varies with the transformation strength. The predictions capture not only the general trends in the bias results, but also show the same dependence of the magnitude of the induced bias with changes in cue transformation.

Although the agreement between the predicted and the actual bias results is quite good, there are a few discrepancies worth pointing out. The actual data show artifacts of the response method (whereby bias tends to be positive for the leftmost position and negative for the rightmost position) that are less evident in the model predictions. In addition, the size of the predicted negative aftereffect is generally larger than the negative aftereffect in the empirical data. This discrepancy may be due to a tendency to readapt to normal cues more quickly than it takes to adapt to the altered cues. On the whole, the data from all five experiments are fit well by the model.

\section{Resolution}

The metric $d_{j}^{\prime}=d^{\prime}\left(\theta_{j+1}, \theta_{j}\right)$ is the resolution with which stimuli $j+1$ and $j$ can be discriminated. The model predicts that $d_{j}^{\prime}$ is given by

$$
d_{j}^{\prime}(r)=\frac{\alpha\left[f_{n}\left(\theta_{j+1}\right)\right]-\alpha\left[f_{n}\left(\theta_{j}\right)\right]}{\sqrt{\beta^{2}+G^{2}\left\{\alpha\left[\frac{\theta_{\text {max }}}{k(r)}\right]-\alpha\left[\frac{\theta_{\text {min }}}{k(r)}\right]\right\}^{2}}} .
$$

Figure 6 plots experimental and predicted values of $d_{j}^{\prime}$ for various runs in the experiments. Again, focusing first on the experimental results, the first test with altered cues yields a large increase in resolution for sources in front of the listener, as compared with the first test with normal cues. However, in all the experimental results, $d^{\prime}$, showed a tendency to decrease as the subjects adapted to the altered cues. A similar decrease is seen in the final (posttraining) test with normal cues, as compared with the initial resolution test with normal cues. In Experiment $\mathrm{F}_{4 b}$, dramatically decreasing the range of stimuli in Run 33 resulted in a large decrease in resolution. There was little effect of this exposure on resolution in Run 37 (using normal cues).

The overall magnitudes of the actual and the predicted results are reasonably close for all five experiments. In the predictions, a similar decrease in resolution is seen with training, but the decrease tends to be smaller than that shown in the experimental results. The decrease in the predictions arises because the effective range increases as subjects adapt, causing an increase in memory noise with training. As in the actual results, the predicted decrease is largest in Experiment $F_{3 \text { mid. }}$. This occurs in the predicted results for two reasons: (1) a smaller range was used in Experiment $F_{3 \text { mid }}$ than in the other experiments, so that the percentage increase in the effective range is largest when changing from altered to normal cues, and (2) the absolute increase in the range with transformed cues is larger for positions closer to the center of the range [both because of the transformation employed and because of the shape of $\alpha(\theta)$ ].

\section{DISCUSSION}

\section{Other Unidimensional Decision Models}

The preliminary model of adaptation is based on the preliminary model of intensity perception of Durlach and Braida (see, e.g., Durlach \& Braida, 1969); however, this is not the only quantitative model of perception of unidimensional stimulus sets. Two other prominent, alternative psychophysical models of resolution are considered here (for a short note reviewing these and other unidimensional stimulus models, see Shiffrin \& Nosofsky, 1994).

Luce and his colleagues (Green, Luce, \& Duncan, 1977; Luce et al., 1976) suggested that intensity resolution changes with stimulus range as a result of shifts in 


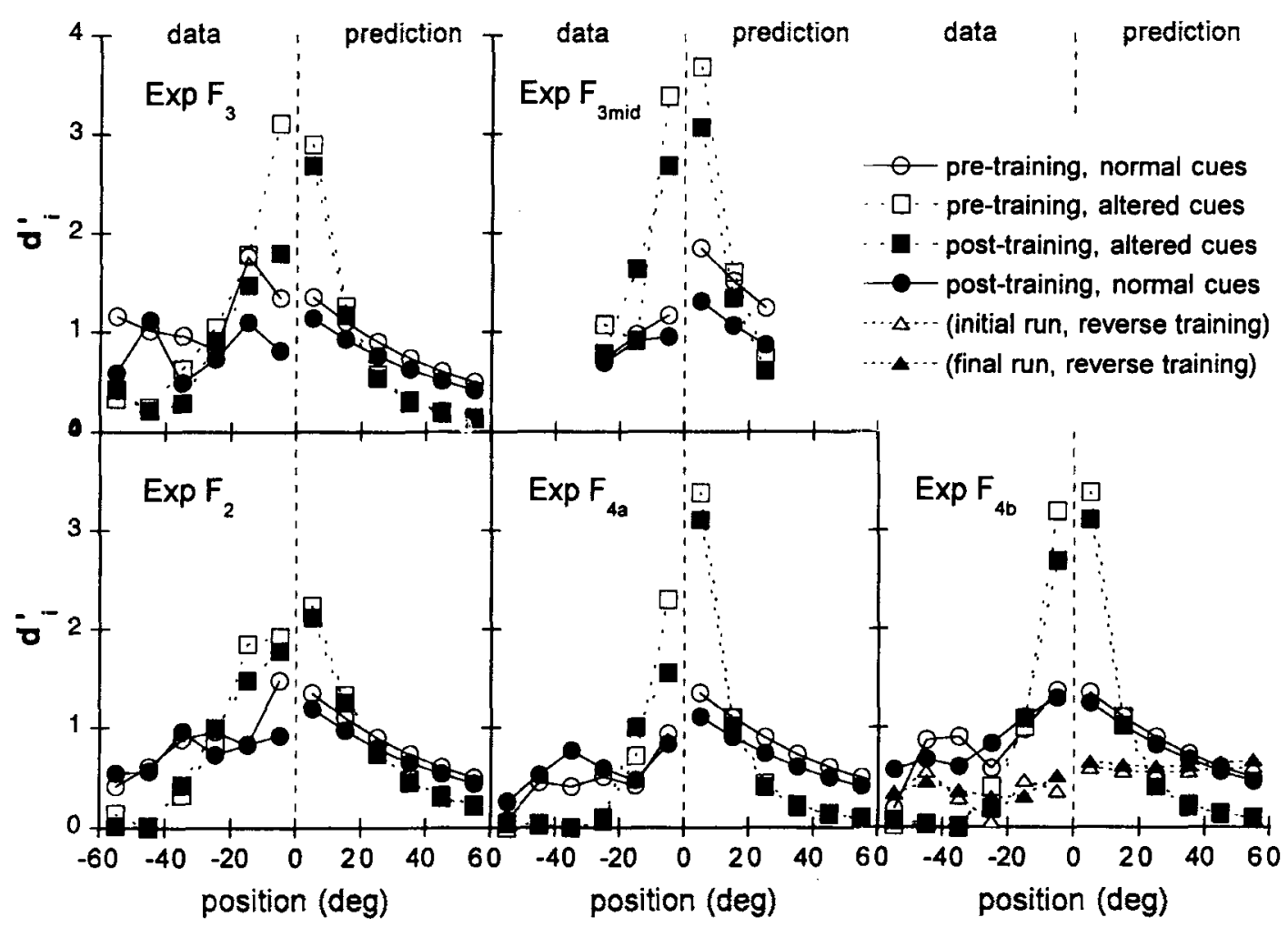

Figure 6. Estimated and predicted resolution $d_{i}^{\prime}$ as a function of position. In each panel, the experimental results, plotted on the left side of each panel, were found by assuming that performance was symmetric about the midline. The predictions from the model are shown on the right side of each panel. To the extent that the left and right halves of each panel are symmetric about the $\boldsymbol{x}$-axis, the model fits the experimental results.

a fixed-width attention band. The posited attention band was assumed to cover a limited range of intensities at any moment. Stimuli with values falling inside the attention band were expected to be resolved more easily than stimuli outside the attention band. The recent history of the stimuli presented to a subject determined the location of the attention band at any point in time. The attentionband model predicts that resolution in small-range intensity experiments is, on average, better than resolution in larger range tasks because a stimulus is more likely to fall inside the attention band. Furthermore, the model predicts that there should be observable sequential effects in resolution, whereby resolution is enhanced when two subsequent stimuli are similar in value (Luce et al., 1982).

A number of investigators have shown that, in a largerange experiment, the value of a preceding stimulus significantly reduces the variability in response to a subsequent stimulus when the two are close in value (Green et al., 1977; Luce et al., 1982; Purks, Callahan, Braida, \& Durlach, 1980; Ward \& Lockhead, 1970). However, analysis suggests that this effect is caused by a shift in response criteria (in a Durlach/Braida-style decision-theory model), rather than by a change in underlying sensitivity (as predicted by the attention-band model; see, e.g., Luce et al., 1982; Purks et al., 1980).
Other experiments demonstrate that, with an appropriate experimental design (for instance, if the stimulus order is controlled so that subsequent presentations are always close in value), underlying sensitivity does improve (Luce et al., 1982; Nosofsky, 1983). Both the attentionband model and the preliminary adaptation model predict that recent history affects resolution. The attentionband model predicts these effects because past history affects the location of the fixed-width attention band. In contrast, the present model posits that the range of stimuli across which subject attention is allocated evolves over the course of tens of trials. In effect, by assuming that the expected range of stimuli determines memory noise, the adaptation model combines elements of the attention-band model with the basic structure of the preliminary model of intensity perception.

Braida and Durlach refined the preliminary model of intensity perception to account for systematic changes in relative intensity resolution with stimulus range. The preliminary model of intensity perception predicts that sensitivity is uniformly scaled up or down with changes in range. However, careful examination of the shape of the cumulative sensitivity functions observed in experiments using different ranges of stimulus intensity reveals that stimulus sensitivity is relatively better at the edges 
of the range than in the middle of the range (Berliner, Durlach, \& Braida, 1977; Luce et al., 1982). Durlach and Braida developed the anchor model of intensity perception to account for this discrepancy (Braida \& Durlach, 1988; Braida et al., 1984). The anchor model assumes that resolution is influenced by the location of perceptual anchors located at the edges of the range of the set of physical stimuli. Judgments of intensity are made by judging the distance between an observed internal decision variable and these internally maintained anchors. Distance judgments for values relatively close to the anchors are more accurate, leading to improved sensitivity for stimuli near the anchors.

Analysis of the cumulative sensitivity function in the present experiments did not reveal any systematic changes in relative sensitivity as a function of the stimulus range (Shinn-Cunningham, 1994). In particular, the results from all the experiments were consistent with the assumptions of the preliminary model of intensity perception. Thus, the simpler, preliminary model was used as the basis for the present adaptation model. Although the results in the present experiments suggest that the range of stimuli used in an experiment does not affect relative sensitivity, this does not necessarily imply that there are not internal anchors or reference positions that are important in auditory spatial perception. One alternative is that spatial perception is qualitatively different from perception of other stimulus dimensions and that any perceptual anchors occur at fixed values, independent of the stimulus range. In particular, since it is important in everyday life to make absolute, egocentric judgments of source location, it is conceivable that subjects constantly maintain a set of fixed anchor locations (such as straight ahead or directly to the left of the head). If spatial resolution is governed by comparisons with such fixed anchors, the observed shape of the cumulative sensitivity function should show little, if any, dependence on the stimulus range. In other words, the present results do not negate the possibility that perceptual anchors are at play in the present experiments. Instead, they suggest that the locations of any spatial perceptual anchors are constant, independent of the experimental paradigm.

\section{What Changes During Spatial Adaptation?}

Even though subjects are trained to associate a finite set of stimulus-response pairs, the model assumes that they do not learn the specific associations used during training. Rather, the model assumes that training causes subjects to alter an internal map relating physical acoustic cues to sound source location. This internal map is constrained: Only linear relationships between stimuli and responses are learned. This assumption is consistent with the ideas of dimension learning (see, e.g., Bedford, 1993) and function learning (see, e.g., Koh \& Meyer, 1991).

In contrast to most rearrangement studies, the work of Bedford (e.g., Bedford, 1993) examined whether subjects can adapt to complex, nonmonotonic remappings. Her results imply that subjects adapt to complex remappings by fitting a roughly linear function to all of the stimulusresponse pairs presented. Mean localization errors persist when the stimulus-response pairs used to train the subjects do not obey a linear relationship. Bedford (1993) demonstrated that training with even a single stimulus location causes subjects to alter their localization responses to sources from many different locations. She interpreted her results as indicating that localization rearrangement experiments cause subjects to alter entire stimulus-response dimensions, rather than learning explicit stimulus-response pairs.

Koh and Meyer (1991) trained subjects to associate pairings of line length (the stimuli) and event duration (the response). Various mathematical relationships governing the trained stimulus-response pairs were tested. The results of the study imply that the easiest mapping to learn is a linear function in log-length log-duration coordinates. Since duration and length perception both obey Weber's law for the visual stimuli used in the experiment, Koh and Meyer concluded that subjects learn most rapidly when presented with stimulus-response functions that are linear in the underlying perceptual dimensions.

The present model and the work by Bedford (1993) also imply that linear stimulus-response relationships are most readily learned; however, both of these studies imply that the stimulus-response mapping is linear in spatial dimensions, not in the internal-decision-axis dimensions. This difference may reflect qualitative differences between learning somewhat arbitrary stimulusresponse relationships (such as that between line length and event duration), and learning spatial-cue-position relationships.

\section{Evidence for Neural Plasticity}

The proposed model of adaptation assumes that peripheral acoustic spatial cues are mapped to some internal perceptual dimension representing source location and that training alters how these peripheral cues are mapped to spatial responses. These assumptions lead naturally to the question of whether neural spatial auditory maps have been observed and whether such maps demonstrate plasticity when sensory stimuli are rearranged. A brief review of these issues is presented below (for a more complete review, see Brainard, 1994; King, 1993; King \& Moore, 1991).

Spatially tuned, topographically organized neurons are observed at the levels of the brainstem through the inferior colliculus and superior colliculus (SC) in a variety of mammals and birds (see, e.g., King, 1993; Knudsen \& Knudsen, 1989). However, as yet there is little evidence for such maps in the cortex of any species, even though spatially sensitive cells are observed (see, e.g., Middlebrooks, 1994).

In normal adult animals, multisensory cells of the SC (or its avian homologue, the optic tectum [OT]) form a topographical map in which visual receptive fields, au- 
ditory receptive fields, and/or somatosensory receptive fields are in registry (see, e.g., King, 1993). The formation of spatial auditory maps in the SC is disrupted when animals are deprived of effective visual or auditory stimulation during a critical period in development (King \& Carlile, 1993; Withington-Wray, Binns, Dhanjal, Brickley, \& Keating, 1990; Withington-Wray, Binns, \& Keating, 1990; Withington, 1992). Similarly, when owls are reared with abnormal visual or binaural cues, the tuning of the auditory map in the OT is altered in a way that compensates for the externally imposed sensory rearrangement (Brainard \& Knudsen, 1995; Knudsen \& Knudsen, 1986, 1989). When the owls are older than the critical period in development, the spatial maps in OT do not completely compensate for the imposed sensory rearrangement (Knudsen \& Knudsen, 1985, 1990). Spatial tuning of OT cells in more mature birds does exhibit some plasticity, especially to less extreme transformations; however, the degree to which spatial tuning of OT cells changes generally decreases with age (Knudsen, Esterly, \& Olsen, 1994; Knudsen \& Knudsen, 1990).

Spatial tuning of cortical neurons also can be affected by sensory experience. In particular, animals deprived of visual stimulation have a larger number of spatially selective auditory neurons with sharper-than-normal spatial tuning (Korte \& Rauschecker, 1993; Rauschecker \& Korte, 1993).

In humans who suffer from congenital aural atresia, the ear canal is blocked or absent, effectively attenuating the sound to that ear by $45-60 \mathrm{~dB}$ (Wilmington, Gray, \& Jahrsdoerfer, 1994). It is possible to correct this anomaly with surgery so that patients receive normal auditory stimulation, but the surgery is usually performed after the head has neared its mature size. Even months after this surgery, patients tend to perform poorly on complex spatial auditory tasks. In contrast, they have normal sensitivity to basic binaural cues, such as interaural time and level differences. One interpretation of this result is that, even though peripherally computed auditory spatial cues are available to these patients, their spatial perception is permanently disrupted by the abnormal sensory stimulation they received during early development.

These studies demonstrate that the spatial tuning of auditory neurons can be affected by long-term sensory rearrangement. This plasticity decreases with age; in particular, without appropriate early experience, spatial auditory perception may be permanently interrupted, even when relatively peripheral auditory processing is intact. Although the demonstrated plasticity is probably important for understanding the development of normal spatial perception, the relationship between these results and the short-term, reversible changes in behavior observed during adaptation to spatial rearrangement of auditory cues is unknown. However, taken together, these results suggest that auditory spatial perception is governed by an internal representation of external space that can be retuned by sensory experience, even in adult animals. The assumptions in the present model are consistent with this view: Short-term adaptation in the model corresponds to a linear retuning of the posited neural representation of auditory space.

\section{Summary}

The preliminary model of adaptation extends the preliminary model of intensity perception (Braida \& Durlach, 1972, 1988; Durlach \& Braida, 1969) by predicting changes in bias and resolution as subjects are trained with remapped auditory localization cues. In particular, the model assumes that the effective stimulus range and the placement of decision criteria are governed by changes in a linearly constrained map relating stimulus to response. The slope of this map evolves over time as subjects adapt, causing the criteria and the effective range (and, hence, the internal noise) to evolve over time. The resulting model predicts that the order of stimulus presentation will affect resolution, an effect that the preliminary intensity perception model did not address.

Decision criteria are assumed to equal the perceivedoptimal criteria in the subjects' decision space. However, since the mapping between physical cue and perceived position (1) changes slowly with time and (2) is constrained to be linear, the model predicts that response bias decreases with exposure time but never disappears. Similarly, the perceived range of positions is always assumed to equal the spatial range of the responses used in the experiments; however, the effective range in the underlying decision space changes as the mapping between perceived and normal-cue position changes. This gradual change in effective range causes a corresponding gradual change in resolution. The model predicts the observed dependence of $\Delta^{\prime}$, bias, and $d^{\prime}$ on stimulus value and on run. Empirical results match predicted values fairly well, both qualitatively and quantitatively.

The model implies that adaptation to rearranged spatial auditory cues is characterized by changes in an internal map that determines how a noisy, internal representation of spatial location is related to positions in external space.

\section{REFERENCES}

BEDFORD, F. L. (1993). Perceptual and cognitive spatial learning. Journal of Experimental Psychology: Human Perception \& Performance, $19,517-530$.

BEDFORD. F. L. (1995). Constraints on perceptual learning: Objects and dimensions. Cognition, 54, 253-297.

Berliner. J. E., Durlach, N. I., \& Braida, L. D. (1977). Intensity perception: VII. Further data on roving-level discrimination and the resolution and bias edge effects. Journal of the Acoustical Society of America, 61, 1577-1585.

Braida. L. D., \& Durlach, N. I. (1972). Intensity perception: II. Resolution in one-interval paradigms. Journal of the Acoustical Society. of America, 51, 483-502.

Braida, L. D., \& Durlach, N. I. (1988). Peripheral and central factors in intensity perception. In G. M. Edelman, W. E. Gall, \& W. M. Cowan (Eds.), Auditory function: Neurobiological bases of hearing (pp. 559-583). New York: Wiley.

Braida, L. D., Lim, J. S., Berliner, J. E., Durlach, N. I., Rabinowitz, W. M., \& PURKS. S. R. (1984). Intensity perception: XIII. Perceptual anchor model of context-coding. Journal of the Acoustical Society of America, 76, 722-731. 
Brainard, M. S. (1994). Neural substrates of sound localization. Current Opinion in Neurobiology, 4, 557-562.

Brainard, M. S., \& Knudsen, E. I. (1995). Dynamics of visually guided auditory plasticity in the optic tectum of the barn owl. Journal of Neurophysiology, 73, 595-614.

Brugge, J. F., Reale, R. A., Hind, J. E., Chan, J. C. K., Musicant, A. D., \& Poon, P. W. F. (1994). Simulation of free-field sound sources and its application to studies of cortical mechanisms of sound localization in the cat. Hearing Research, 73, 67-84.

CARLILE, S. (1996). Virtual auditory space: Generation and applications. New York: RG Landes.

DAY, R. H., \& Singer, G. (1967). Sensory adaptation and behavioral compensation with spatially transformed vision and hearing. Psychological Bulletin, 67, 307-322.

Durlach, N. I., \& Braida, L. D. (1969). Intensity perception: I. Preliminary theory of intensity resolution. Journal of the Acoustical Society of America, 46, 372-383.

Durlach, N. I., Shinn-Cunningham, B. G., \& Held, R. M. (1993). Supernormal auditory localization: I. General background. Presence, 2, 89-103.

EBENHOLTZ, S. M. (1970). Temporal characteristics of a comparator in adaptation to optical tilt. Perception \& Psychophysics, 7, 365-366.

EBENHOLTZ, S. M. (1973). Optimal input rates for tilt adaptation. American Journal of Psychology, 86, 193-200.

GreEn, D. M., LuCE, R. D., \& DUNCAN, J. E. (1977). Variability and sequential effects in magnitude production and estimation of auditory intensity. Perception \& Psychophysics, 22, 450-456.

KaliL, R., \& Freedman, S. J. (1967). Compensation for auditory rearrangement in the absence of observer movement. Perceptual \& Motor Skills, 24, 475-478.

KING, A. J. (1993). A map of auditory space in the mammalian brain: Neural computation and development. Experimental Physiology, 78, 559-590.

KING, A. J., \& CARLILE, S. (1993). Changes induced in the representation of auditory space in the superior colliculus by rearing ferrets with binocular eyelid suture. Experimental Brain Research, 94, 444455.

KING, A. J., \& Moore, D. R. (1991). Plasticity of auditory maps in the brain. Trends in Neurosciences, 14, 31-37.

KNUdSEN, E. I., Esterly, S. D., \& OlSEN, J. F. (1994). Adaptive plasticity of the auditory space map in the optic tectum of adult and baby barn owls in response to external ear modification. Journal of Neurophysiology, 71, 79-94.

KNUDSEN, E. I., \& KNUDSEN, P. F. (1985). Vision guides the adjustment of auditory localization in young barn owls. Science, 230, 545-548.

KNUDSEN, E. I., \& KNUDSEN, P. F. (1986). The sensitive period for auditory localization in barn owls is limited by age, not by experience. Journal of Neuroscience, 6, 1918-1924.

KNUdSEN, E. I., \& KNUDSEN, P. F. (1989). Vision calibrates sound localization in developing barn owls. Journal of Neuroscience, 9, 33063313.

KNUDSEN, E. I., \& KNUdSEN, P. F. (1990). Sensitive and critical periods for visual calibration of sound localization by barn owls. Journal of Neuroscience, 10, 222-232.

KoEhNKe, J., \& Durlach, N. I. (1989). Range effects in the identification of lateral position. Journal of the Acoustical Society of America, 86, 1176-1178.

KOH, K., \& MEYER, D. E. (1991). Function learning: Induction of continuous stimulus-response relations. Journal of Experimental Psy. chology: Learning, Memory, \& Cognition, 17, 811-836.

KORTE, M.. \& RAusCHECKER, J. P. (1993). Auditory spatial tuning of cortical neurons is sharpened in cats with early blindness. Journal of Neurophysiology, 70, 1717-1721.

LuCE, R. D., GREEN, D. M., \& WEBER, D. L. (1976). Attention bands in absolute identification. Perception \& Psychophysics, 20, 49-54.

Luce, R. D., Nosofsky, R. M., Green, D. M., \& Smith, A. F. (1982). The bow and sequential effects in absolute identification. Perception \& Psychophysics, 32, 397-408.

MaCmillan, N. A. (1987). Beyond the categorical/continuous distinc- tion: A psychophysical approach to processing modes. In S. Harnad (Ed.), Categorical perception: The groundwork of cognition (pp. 5385). New York: Cambridge University Press.

Macmillan, N. A., Braida. L. D., \& GoldberG, R. F. (1987). Central and peripheral processes in the perception of speech and nonspeech sounds. In M. E. H. Schouten (Ed.), The psychophysics of speech perception (pp. 28-45). Dordrecht: Martinus Nijhoff.

Macmillan, N. A., Goldberg, R. F., \& Braida, L. D. (1988). Resolution for speech sounds: Basic sensitivity and context memory on vowel and consonant continua. Journal of the Acoustical Society of America, 84, 1262-1280.

MIDDLEBROOKS, J. (1994). A panoramic code for sound location by cortical neurons. Science, 264, 842-844.

Mikaelian, H. H. (1969). Adaptation to rearranged ear-hand coordination. Perceptual \& Motor Skills, 28, 147-150.

Mikaelian, H. H. (1972). Lack of bilateral generalization of adaptation to auditory rearrangement. Perception \& Psychophysics, 11, 222-224.

Mikaelian, H. H. (1974). Adaptation to displaced hearing: A nonproprioceptive change. Journal of Experimental Psychology, 103, 326-330.

MILLS, A. W. (1958). On the minimum audible angle. Journal of the Acoustical Society of America, 30, 237-246.

Nosorsky, R. M. (1983). Shifts of attention in the identification and discrimination of intensity. Perception \& Psychophysics, 33, 103-1 12.

Purks, S. R., Callahan, D. J., Braida, L. D., \& Durlach, N. I. (1980). Intensity perception: X. Effect of preceding stimulus on identification performance. Journal of the Acoustical Society of America, 67, 634-637.

RAUSCHECKER, J. P., \& KoRTE, M. (1993). Auditory compensation for early blindness in cat cerebral cortex. Journal of Neuroscience, 13, 4538-4548.

Recanzone, G. H. (1998). Rapidly induced auditory plasticity: The ventriloquism aftereffect. Proceedings of the National Academy of Sciences, 95, 869-875.

Searle, C. L., Braida, L. D., Davis, M. F., \& Colburn, H. S. (1976). Model for auditory localization. Journal of the Acoustical Society of America, 60, 1164-1175.

SHIFFrin, R. M., \& Nosofsky, R. M. (1994). Seven plus or minus two: A commentary on capacity limitation. Psychological Review, 101, 357-361.

Shinn-Cunningham, B. G. (1994). Adaptation to supernormal auditory localization cues in an auditory virtual environment. Unpublished doctoral dissertation, Massachusetts Institute of Technology.

Shinn-Cunningham, B. G., Durlach, N. I., \& Held, R. M. (1998a). Adapting to supernormal auditory localization cues: I. Bias and resolution. Journal of the Acoustical Society of America, 103, 3656-3666.

Shinn-Cunningham, B. G., Durlach, N. 1.. \& Held, R. M. (1998b). Adapting to supernormal auditory localization cues: II. Constraints on adaptation of mean response. Journal of the Acoustical Soriety of America, 103, 3667-3676.

Shinn-Cunningham, B. G., Lehnert, H., Kramer, G., Wenzel, E. M., \& Durlach, N. I. (1997). Auditory displays. In R. Gilkey \& T. Anderson (Eds.), Binaural and spatial hearing in real and virtual environments (pp. 611-663). Hillsdale, NJ: Erlbaum.

WARD, L. M. \& LockHEAD, G. R. (1970). Sequential effects and memory in categorical judgement. Journal of Experimental Psychology, 17, 27-34.

WELCH, R. B. (1978). Perceptual modification: Adapting to altered sensory environments. New York: Academic Press.

Welch, R. B., \& WARREN, D. H. (1980). Immediate perceptual response to intersensory discrepancy. Psychological Bulletin, 88, 638667.

WENZEL, E. M. (1992). Localization in virtual acoustic displays. Presence, 1, 80-107.

Wightman, F. L., \& KISTLER, D. J. (1997). Monaural sound localization revisited. Journal of the Acoustical Society of America, 101 , 1050-1063.

Willey, C. F., Inglis, E., \& Pearce, C. H. (1937). Reversal of auditory localization. Journal of Experimental Psychologv, 20, 114-130.

Wilmington, D., Gray, L., \& JAhrSDoerFer. R. (1994). Binaural pro- 
cessing after corrected congenital unilateral conductive hearing loss. Hearing Research, 74, 99-114.

WiTHINGTON, D. J. (1992). The effect of binocular lid suture on auditory responses in the guinea-pig superior colliculus. Neuroscience Letters, 136, 153-156.

Withington-Wray, D. J., Binns, K. E., Dhanjal, S. S., Brickley, S. G., \& KEATING, M. J. (1990). The maturation of the superior collicular map of auditory space in the guinea pig is disrupted by developmental auditory deprivation. European Journal of Neuroscience, 2, 693-703.

Withington-Wray, D. J., Binns, K. E., \& Keating, M. J. (1990). The maturation of the superior collicular map of auditory space in the guinea pig is disrupted by developmental visual deprivation. European Journal of Neuroscience, 2, 682-692.

YounG, P. T. (1928). Auditory localization with acoustic transposition of the ears. Journal of Experimental Psychology, 11, 399-429.

\section{NOTES}

1. Note that the model assumes that $\Delta^{\prime}$ equals the sum of the distances between adjacent stimuli in the set, measured in units of standard devi- ation. Thus, $\Delta^{\prime}$ equals the sum over all adjacent stimulus pairs of $d_{j}^{\prime}$, the standard psychophysical measure of stimulus resolution. The measure $d_{j}^{\prime}$ is considered in detail below.

2 . In the present paper, bias is denoted by $B$ (rather than by the more usual symbol $\beta$ ) to avoid confusion with the sensation noise in the model.

3. Bias estimates and resolution estimates (shown in Figures 4, 5, and 6) were found using a maximum likelihood estimation approach detailed in Shinn-Cunningham et al. (1998a).

4. Theoretically, the optimal placement for all criteria should be farther from the zero-azimuth location than are the actual criteria placement for naive subjects; however, bias in the first altered-cue run tends to be positive for the leftmost criterion and negative for the rightmost criterion in all the experiments. This is an artifact of the identification method, reflecting the fact that subjects cannot accurately indicate the heard location of a source when it falls outside the range of allowed responses.

(Manuscript received April 18, 1997; revision accepted for publication September 17, 1998.)

\section{Associate Editor Changes for Perception \& Psychophysics}

We announce with regret the resignation of Associate Editor Steven Yantis. Professor Yantis has generously provided Perception \& Psychophysics with two years of his time and expertise.

At the same time, it is a pleasure to welcome Kimron Shapiro of the University of Wales, Bangor, as a new associate editor. Professor Shapiro can be reached at k.shapiro@bangor.ac.uk. 